\title{
Interstitial Fibrosis of Unilateral Ureteral Obstruction is Exacerbated in Kidneys of Mice Lacking the Gene for Inducible Nitric Oxide Synthase
}

\author{
David Hochberg, Christopher W. Johnson, Jie Chen, Drorit Cohen, Joshua Stern, \\ E. Darracott Vaughan, Jr., Dix Poppas, and Diane Felsen
}

Center for Pediatric Urology and Laboratory for Minimally Invasive Urologic Surgery, Department of Urology, Children's Hospital of New York, Weill Medical College of Cornell University, New York, New York

SUMMARY: Unilateral ureteral obstruction (UUO) is characterized by decreases in renal function and increases in interstitial fibrosis. Previous studies have indicated that pharmacologic manipulations that increase nitric oxide (NO) are beneficial to the obstructed kidneys. NO is produced from arginine by nitric oxide synthase (NOS), an enzyme that exists in both constitutive and inducible (iNOS) forms. To determine the role of the inducible form of NOS in UUO, we used mice with a targeted deletion of iNOS (iNOS -/- mice) and compared them with wild-type (WT) mice. Kidneys were obstructed for 2 weeks in both WT and iNOS -/- mice, and were then removed and bisected. Half of the kidney was embedded in paraffin and tissue sections were examined for interstitial volume or the presence of macrophages. The remainder was flash-frozen and samples were used to measure tissue collagen (hydroxyproline) or transforming growth factor- $\beta$ (TGF- $\beta$ ). This study demonstrates that both cortex and medulla of obstructed kidneys of iNOS -/- mice exhibit significantly increased interstitial volume and interstitial macrophages as compared with their WT counterparts. Furthermore tissue collagen was increased to $9.2 \pm 1.3 \mu \mathrm{g} / \mathrm{mg}$ tissue in WT obstructed kidneys, whereas in iNOS -/- kidneys, collagen was increased to $13.2 \pm 0.8 \mu \mathrm{g} / \mathrm{mg}$ tissue. The profibrotic cytokine TGF- $\beta$ was also significantly increased in obstructed kidneys of iNOS -/- mice, as compared with WT mice. No differences were noted between the unobstructed kidneys of iNOS -/- mice compared with WT mice in any of the parameters examined. These results demonstrate that targeted deletion of the iNOS results in exacerbation of fibrotic events in the obstructed kidney. These results confirm previous pharmacologic studies, and suggest that NO produced via the inducible NOS normally serves a protective function in UUO. (Lab Invest 2000, 80:1721-1728).

$R$ enal interstitial fibrosis has been proven to be a histologic hallmark of chronic ureteral obstruction (Gulmi et al, 1998; Klahr and Morrissey, 1998). Characteristic findings include the expansion of the renal tubular interstitium with infiltration of monocytes and macrophages. This is accompanied by the accumulation of both normal and abnormal interstitial matrix proteins, and increases in tissue transforming growth factor- $\beta$ (TGF- $\beta$ ) (Diamond et al 1994; Sharma et al, 1993; Wright et al, 1996). Presumably, fibrosis develops as an imbalance between interstitial protein synthesis, deposition, and degradation (Border and Noble, 1994; Eddy, 1996).

Nitric oxide (NO) has been implicated as a mediator, messenger, or regulator of cell function in physiologic situations as diverse as platelet function, vascular tone, memory, hepatocyte function, apoptosis, and penile erectile function (Bhagat and Vallance, 1996; Kim et al, 1999; Kone, 1997). NO is synthesized from its precursor, L-arginine via the enzyme NO synthase

Received July 12, 2000.

Address reprint requests to: Dr. Diane Felsen, Associate Research Professor of Pharmacology in Urology, Weill Medical College of Cornell University, Department of Urology, Box 94, 1300 York Avenue, New York, New York 10021. Fax: $2127463314 ;$ E-mail:dfelsen@med.cornell.edu
(NOS), which exists in three forms. Neuronal NOS (nNOS) and endothelial NOS (eNOS) are constitutively expressed, and there is an inducible isoform (iNOS). When NOS is induced, iNOS produces large quantities of NO, which has been implicated in the host response to sepsis and infection, hemorrhagic shock, and other disease models (Hierholzer et al, 1998; Kone, 1997).

Because of the abundance of physiologic and pathophysiologic roles in which NO participates, the manipulation of NO offers therapeutic potential in a variety of disease states. Pharmacologic agents that inhibit NOS can decrease the generation of NO. Selective inhibitors of iNOS commonly used experimentally include aminoguanidine and L-N6-(1iminoethyl)lysine (Bryk and Wolff, 1998; Moore et al, 1994). Newer mechanism-based inactivators of NOS, which require catalytic turnover to irreversibly inactivate NOS, have been described; in addition, allosteric inhibitors of NOS have been identified by combinatorial chemistry (Bryk and Wolff, 1999; McMillan et al, 2000). At issue with the use of NOS inhibitors in vivo is not only the cross-reactivity between the various forms of NOS, but also the pharmacologic side effects of these agents.

It has been recently recognized that increased NO generation is a common characteristic of certain pharmacologic agents that ameliorate interstitial fibrosis. 
The administration of arginine and angiotensin converting enzyme (ACE) inhibitors have both been found to blunt the fibrotic response to unilateral ureteral obstruction (UUO) (Ishidoya et al, 1995; Morrissey et al, 1996). Arginine is a direct precursor to NO, whereas ACE inhibitors are thought to augment NO production indirectly by stimulating bradykinin production (Zhang et al, 2000). Comparable evidence also exists in that the pharmacologic inhibition of NOS during UUO exacerbates the resulting interstitial fibrosis (Morrissey et al, 1996). Thus NO appears to be protective, but the molecular source of the $\mathrm{NO}$ is unknown. To avoid some of the difficulties associated with pharmacologic inhibition of $\mathrm{NO}$, and to more specifically evaluate the role of iNOS in the fibrosis that results from UUO, we have used mice with a targeted deletion of the iNOS gene (iNOS -/- mice) to study interstitial fibrosis in UUO.

\section{Results}

\section{Histologic Analysis}

In the present study we compared the response to UUO in both control unobstructed kidneys and in obstructed kidney from both wild-type (WT) and iNOS
-/- mice. In Figure 1 are shown trichrome stained sections from these kidneys. In Figure 1, a and b, are shown the unobstructed kidneys of WT (Fig. 1a) and iNOS -/- mice (Fig. 1b). Kidneys from both types of mice show a regular arrangement of tubules with little interstitial fibrosis. There is little apparent difference between kidneys from WT and iNOS -/- mice. UUO is accompanied by tubular dilatation, associated with a marked increase in interstitial collagen deposition (blue staining) in both WT kidneys (Fig. 1c) and iNOS -/- kidneys (Fig. 1d) (compare Fig. 1a with b, and c with d). The kidneys of obstructed iNOS -/- mice exhibited increased interstitial volume, interstitial fibrosis, and interstitial cellularity as compared with WT mice (compare Fig. 1c with d), as will be detailed and quantified below.

\section{Interstitial Volume}

Interstitial volume expansion in response to UUO has previously been shown in both rat and rabbit kidney (Sharma et al, 1993; Wright et al, 1996). In the present study we confirm that a similar change occurs in the mouse kidney. In WT mice there was a significant increase (2.5 times compared with control) in cortical
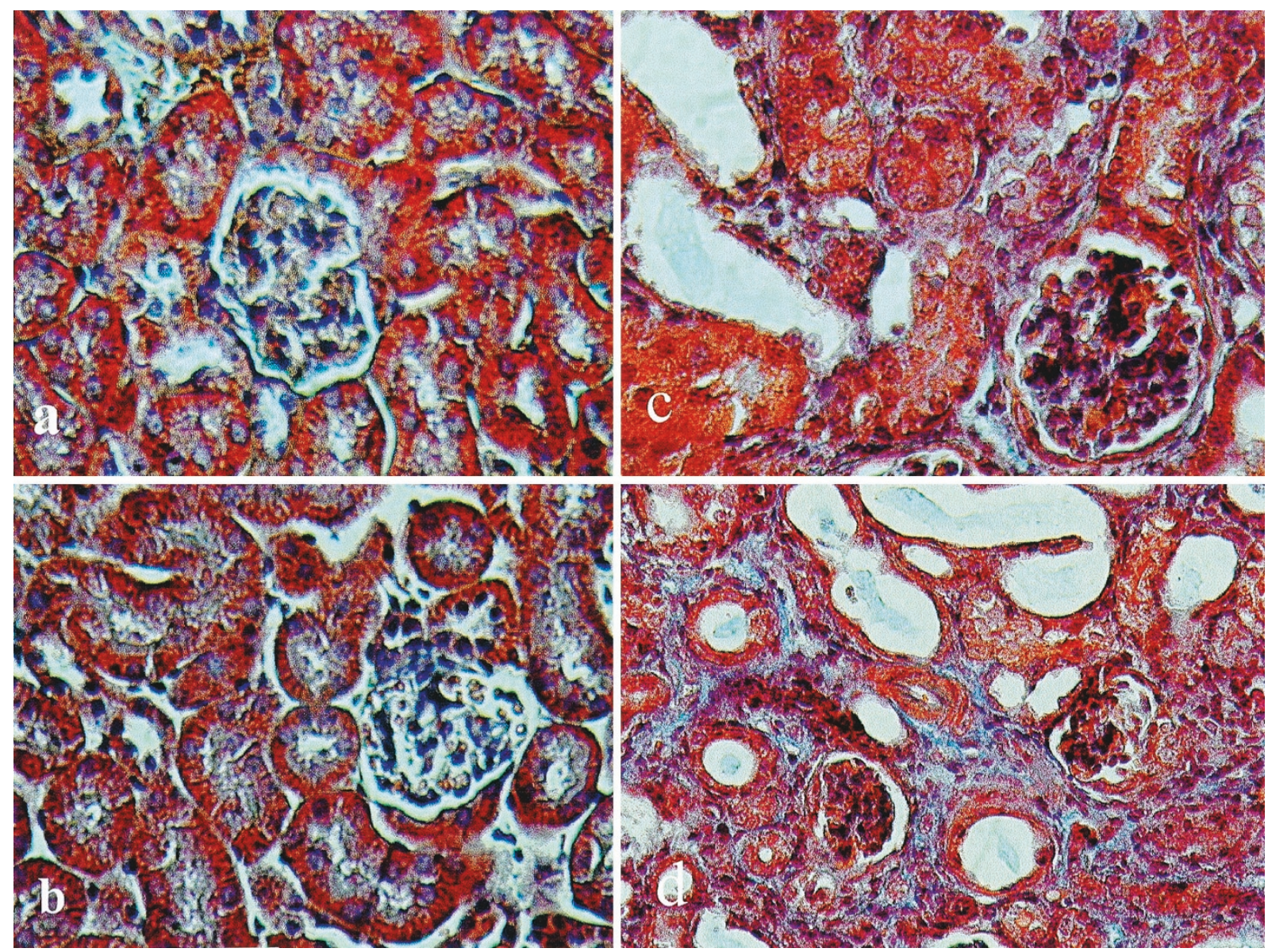

\section{Figure 1.}

Masson's trichrome staining of mouse kidney sections. Paraffin sections, $5-\mu \mathrm{m}$ thick, were stained with Masson's trichrome. The blue-appearing collagen is particularly evident in obstructed specimens. a, Control wild-type(WT) kidney; $b$, control inducible nitric oxide synthase (iNOS) -/- kidney; $c$, 14-day obstructed WT kidney; $d$, 14-day obstructed iNOS -/- kidney. Original magnification, $\times 400$. 
interstitial volume at 14 days of obstruction (Table 1). Medullary interstitial volume was also significantly increased.

We found that the interstitial volume expansion was significantly greater $(p<0.05)$ in both the cortex and medulla of obstructed iNOS -/- kidneys compared with the obstructed WT (Table 1). After obstruction, cortical interstitial volume increased 3.2-fold in kidneys of iNOS -/- mice as compared with a 2.5-fold increase in kidneys of WT mice.

No significant difference, quantitative or qualitative, was appreciated between control WT and iNOS -/kidneys. Control WT cortical interstitial volume (\%) was $11.4 \pm 1.9$, whereas control iNOS -/- interstitial volume was $11.1 \pm 0.8$ (Table 1$)$. No differences were detected in the medulla (Table 1).

\section{Tissue Collagen}

Tissue collagen, as measured by analyzing tissue hydroxyproline (see "Methods") was also assessed in kidneys of WT and iNOS-/- mice. At 14 days there were significant differences in the collagen content of the obstructed WT and iNOS -/- kidneys as compared with their respective control kidneys (Table 2). Furthermore the obstructed iNOS -/- kidney contained more collagen than the obstructed WT kidney $(13.1 \pm 0.8$ compared with $9.2 \pm 1.3 \mu \mathrm{g} / \mathrm{mg}$ tissue dry weight; Table 2).

\section{Tissue TGF- $\beta$}

Since TGF- $\beta$ has been considered to be a profibrotic cytokine with a role in UUO, we examined tissue TGF- $\beta$ using the mink lung bioassay. In WT mice at 14 days, tissue TGF- $\beta$ was increased by 6.8 -fold in the obstructed kidney relative to its control(Table 2). In comparison, there was more than a 28 -fold increase in tissue TGF- $\beta$ with obstruction in iNOS -/- mice. Tissue TGF- $\beta$ in the obstructed kidney of iNOS -/- mice was significantly greater than that in obstructed kidney of WT mice.

\section{Macrophage Quantification}

An increase in interstitial cellularity in particular monocytes and macrophages is one of the primary features of interstitial fibrosis. To identify macrophages we used a monoclonal antibody to the F4/80 antigen, a murine macrophage-specific cellular membrane marker that maintains its antigenicity during paraformaldehyde fixation and paraffin embedding (Gordon et al, 1992).

WT and iNOS -/- mice both showed significant increases in F4/80-positive cells after 14 days of obstruction (Table 3). The number of F4/80-positive cells per high power field increased by 20.9 and 26.0 cells in the cortex and medulla of the WT kidney, respectively. Increases in iNOS -/- cortical and medullary F4/80-positive cells were 34.2 and 41.4 cells per high power field, respectively.

Furthermore the number of F4/80-positive cells was found to be significantly greater in both cortex and medulla of obstructed kidneys of iNOS -/- mice compared with WT (Table 3). Comparison of control WT with iNOS -/- kidneys illustrated no significant differences.

\section{Discussion}

Interstitial fibrosis includes the accumulation of interstitial matrix proteins, inflammatory cell infiltrates, and the production of proinflammatory cytokines that leads to a complex and dynamic imbalance among interstitial matrix synthesis, deposition, and degradation. Interstitial fibrosis is the histologic hallmark of virtually all progressive renal diseases, including chronic ureteral obstruction, and it is the single best histologic correlate of decline in renal function and long-term prognosis (Eddy, 1996). Although previous studies have implicated a role for NO in ameliorating fibrosis during UUO, the enzymatic source of NO has not been determined. The present study used iNOS -/mice to probe the role of inducible NOS in this process. Our results demonstrate that interstitial fibrosis is exacerbated in both the cortex and medulla of iNOS -/- mice after 14 days UUO compared with WT mice. UUO in iNOS -/- mice is accompanied both by significantly greater increases in interstitial volume, tissue collagen, and TGF- $\beta$ and by interstitial macrophage infiltration. This suggests that the NO synthesized by the inducible form of NOS, may normally be protective in UUO.

Previous investigators have suggested roles for NO in models of fibrosis in other systems. By using L-NAME in the drinking water of rats, the chronic inhibition of NOS has been shown to increase angiotensin II-induced cardiac fibrosis (Hou et al, 1995). Furthermore the chronic treatment of rats with L-NAME has been shown to result in both myocardial and perivascular fibrosis (Numaguchi et al, 1995). In contrast other authors have been unable to confirm these findings using similar models (Sigusch et al, 1996).

In the kidney the role of $\mathrm{NO}$ in models of ureteral obstruction and other diseases is complex. The involvement of NO has often been studied using arginine supplementation. Use of arginine administration (either through dietary supplement or by injection) has

Table 1. Interstitial Volume (\%) in iNOS -/- and Wild-Type (WT) Mice at 14 Days of UUO

\begin{tabular}{ccccc}
\hline & WT control & WT-obstructed & iNOS $-/$ - control & iNOS $-/-$ obstructed \\
\hline Cortex & $11.4 \pm 1.9$ & $28.7 \pm 3.3^{*}$ & $11.1 \pm 0.8$ & $35.4 \pm 2.1^{*} \ddagger$ \\
Medulla & $15.8 \pm 2.3$ & $32.7 \pm 1.5^{*}$ & $18.5 \pm 1.2$ & $43.7 \pm 1.5^{\star} \ddagger$ \\
\hline
\end{tabular}

* $p<0.05$ compared to unobstructed control; $\neq p<0.05$ compared with WT obstructed. 
Table 2. Tissue Collagen and Tissue TGF- $\beta$ in iNOS $-/-$ and WT Mice ${ }^{a}$

\begin{tabular}{lcccc}
\hline & WT control & WT-obstructed & iNOS $-/$ - control & iNOS $-/-$ obstructed \\
\hline Collagen & $4.6 \pm 0.5$ & $9.2 \pm 1.3^{*}$ & $7.8 \pm 0.7$ & $13.2 \pm 0.8^{*} \ddagger$ \\
TGF- $\beta$ & $2.4 \pm 0.3$ & $16.5 \pm 1.0^{*}$ & $1.6 \pm 0.9$ & $45.0 \pm 3.6^{*} \ddagger$ \\
\hline
\end{tabular}

* $p<0.05$ compared to unobstructed control; $\ddagger p<0.05$ compared with WT obstructed.

${ }^{a}$ Collagen, $\mu \mathrm{g} / \mathrm{mg}$ tissue, dry weight; TGF- $\beta, \mathrm{pg} / \mathrm{mg}$ tissue, wet weight.

Table 3. F4/80-Positive Cells per High Power Field

\begin{tabular}{ccccc}
\hline & WT control & WT-obstructed & iNOS $-/-$ control & iNOS $-/-$ obstructed \\
\hline Cortex & $0.5 \pm 0.2$ & $21.4 \pm 0.9^{*}$ & $1.7 \pm 0.9$ & $35.9 \pm 1.3^{*} \ddagger$ \\
Medulla & $1.2 \pm 1.0$ & $27.2 \pm 6.0^{*}$ & $2.9 \pm 0.3$ & $44.3 \pm 1.7^{\star} \ddagger$ \\
\hline
\end{tabular}

${ }^{*} p<0.05$ compared with unobstructed control; $\ddagger p<0.05$ compared with WT obstructed.

resulted in amelioration of fibrosis in models of subtotal nephrectomy, diabetes, hypertensive nephrosclerosis, and cyclosporine nephrotoxicity (Chen et al, 1993; De Nicola et al, 1993; Katoh et al, 1994; Reyes et al, 1993). In glomerulonephritis, arginine supplementation has both ameliorated and exacerbated the disease (Peters et al, 1999, 2000). The timing of administration of arginine may be a critical factor in its effects. Beneficial effects of the administered arginine may result from its metabolism to NO. The resulting NO may by itself be antifibrotic (Trachtman et al, 1996). In addition metabolism of arginine to NO by endothelial NOS may result in reversal of vasoconstrictive effects caused by previously decreased arginine. In contrast detrimental effects of arginine administration may be caused by synthesis of large quantities of $\mathrm{NO}$ or other toxic metabolites resulting from metabolism of arginine by iNOS, which could be responsible for tissue damage. In addition the possibility that arginine supplementation results in metabolism of arginine to non-NO products also needs to be considered in assessing both beneficial and harmful effects of arginine supplementation.

In UUO specifically, several studies have demonstrated a protective role for NO. To pharmacologically increase the production of $\mathrm{NO}$, rats were treated with ACE inhibitors, or a dietary supplement of L-arginine. Both of these agents were confirmed to increase NO generation, as demonstrated by increases in urinary nitrite concentration. It was shown that experimentally obstructed rats given each of these agents displayed significantly less interstitial fibrosis after UUO. Furthermore the pharmacologic inhibition of NOS with L-NAME not only reversed the beneficial effects when used with the aforementioned agents, but when used alone, the interstitial fibrosis of UUO was significantly exacerbated (Morrissey et al, 1996). Low levels of arginine have been found associated with bilateral ureteral obstruction (Reyes et al, 1994); in studies on the effects of arginine on renal blood flow in a canine model of UUO, we found that decreased renal function in UUO may be related to a decreased tissue supply of arginine (Schulsinger et al, 1997). Furthermore, in a study examining the unilateral release of obstruction in a model of bilateral ureteral obstruction, arginine ad- ministration has been shown to enhance recovery of renal function (Reyes et al, 1992). The present study is in agreement with previous reports in that we demonstrate an exacerbation of fibrosis when the gene for iNOS is deleted. The general protective role of NO in UUO is also supported by other studies from this laboratory in which the apoptosis of UUO is significantly exaggerated in iNOS -/- mice (Miyajima et al, 2000b).

In contrast, the present results, and those cited above using pharmacologic manipulation of $\mathrm{NO}$, are in conflict with those recently reported by Huang et al (2000). Using iNOS -/- mice with UUO, they reported decreased fibrosis as assessed by trichrome staining and collagen I deposition. In addition, the cortex of kidneys of iNOS -/- mice with UUO was thinner than the cortex of WT mice with UUO, suggesting that cortical thinning is associated with decreased fibrosis. However, using MRI in patients it has been shown that the renal cortex of patients with interstitial fibrosis is thinner than cortex of normal controls (Kettritz et al, 1996), which is contradictory to the finding of Huang et al. It is unclear at present why there are discrepancies between these studies.

The majority of the studies of $\mathrm{NO}$ in models of fibrosis suggest that NO is antifibrotic, and conversely that inhibition of $\mathrm{NO}$ is profibrotic (Hou et al, 1995; Morrissey et al, 1996; Tomita et al, 1998). Because of the lack of specificity of the pharmacologic NOS inhibitors used in these studies, a clear determination of the impact of each specific NO synthase in UUO has yet to be illustrated. Thus the present study, using iNOS -/- mice, not only confirms that a lack of NO is associated with exacerbation of fibrosis, but it also serves to implicate the inducible form of NOS in this process. Studies using iNOS -/- mice are thus able to establish a role for the inducible NOS, which can only be suggested using pharmacologic studies. This has been shown in several different disease models (Mashimo and Goyal, 1999).

Furthermore, unlike previous studies using iNOS inhibitors (Morrissey et al, 1996), we do not appreciate any significant differences between the iNOS -/- and WT control kidneys. Neither histologic appearance nor 
tissue collagen or interstitial volume are different in iNOS -/- as compared with WT mice. This suggests that previous differences found by using pharmacologic inhibition of NOS may be attributed to the effects of these agents on the constitutively expressed NO synthases, or to other nonspecific effects of these drugs.

Results of tissue TGF- $\beta$ analysis confirm that the obstructed kidney contained increased TGF- $\beta$ as compared with the control kidney, as has been previously shown in rats (Wright et al, 1996). Furthermore, the TGF- $\beta$ is further increased in iNOS -/- mice. This finding is in agreement with previous studies using L-NAME treatment of rats in a cardiac fibrosis model. In that model, treatment with L-NAME leads to increased expression of TGF- $\beta$ and extracellular matrix proteins after L-NAME administration (Tomita et al, 1998). Another striking finding is the significantly greater increase in macrophage infiltration in the iNOS -/- mice. In vivo, NO has been shown to inhibit leukocyte adhesion to the endothelium of blood vessels (Kanwar and Kubes, 1995). Conversely, the inhibition of NOS has been shown to elicit significant leukocyte adhesion (Akimitsu et al, 1995). In vitro, inhibition of NO with pharmacologic NOS inhibitors up-regulates the mRNA of the monocyte chemoattractant protein, MCP-1, which plays a role in monocyte recruitment (Zeiher et al, 1995). Recent studies using iNOS -/- mice have demonstrated similar findings in two different colitis models. In both the TNB and DSS models of colitis, iNOS -/- show increased severity and greater damage as compared with WT animals. These results support a role for iNOS in protecting against inflammation (Beck et al, 1999; Qiu et al, 1998).

The mechanism by which interstitial fibrosis causes compromise of renal function is not clearly understood. A significant factor is likely to be the ultimate obliteration of the renal tubules by the fibrogenic process (Eddy, 1996). Also, destruction of postglomerular interstitial capillaries, as well as the overexpression of vasoconstrictors, is likely to compromise the oxygen supply to the interstitium, thus worsening renal function (Eddy, 1996). This may help to explain the importance of NO, especially its vasodilatory effects, during chronic ureteral obstruction.

In summary, our data demonstrate that interstitial fibrosis is exacerbated in the obstructed kidneys of mice lacking the gene for inducible NO synthase. iNOS -/- mice showed evidence of significantly greater interstitial fibrosis after UUO, as assessed by increases in interstitial volume, collagen, TGF- $\beta$, and macrophage infiltration. These data therefore suggest that, among its myriad of pathophysiologic functions, inducible NO serves a protective role during chronic UUO. With the advent of newer, more specific pharmacologic manipulation of $\mathrm{NO}$, strategies that augment the generation of nitric oxide may soon prove beneficial as antifibrotic agents during chronic ureteral obstruction.

\section{Materials and Methods}

iNOS -/- Mice

Mice with a targeted deletion of the iNOS gene were obtained from Dr. Carl Nathan, Cornell University Medical College. The iNOS gene was inactivated through homologous recombination using a replacement vector targeted to delete the proximal 585 bases of the iNOS promoter. The extent and selectivity of iNOS deficiency in vivo were confirmed by Northern and Southern blots. Southern blots demonstrated a single iNOS allele in WT animals, which was not detected in iNOS -/- mice. iNOS was unable to be induced, whereas eNOS and nNOS expression was neither diminished nor increased in compensation for iNOS deficiency (MacMicking et al, 1995).

\section{Tail Clip DNA and PCR}

In the present study, the NOS genotype was determined by PCR using tail clip DNA. Tails were cut into 0.4-0.6-cm pieces and DNA isolated using a QIAamp kit from Qiagen (Valencia, California). Briefly, samples were incubated in Proteinase $\mathrm{K}$, centrifuged, and incubated in buffer containing ethanol. RNAse was added to the sample, which was then spun on a QIAamp spin column.

PCR was carried out as described (MacMicking et al, 1995) for 35 cycles with Taq polymerase $(0.75 \mathrm{U})$ at an annealing temperature of $60^{\circ} \mathrm{C}$ (30 seconds) with primer extension at $72^{\circ} \mathrm{C}$ (3.0 minutes). Seventy nanograms of DNA was used per reaction, and primers were specific for either WT iNOS (PCR product, $413 \mathrm{bp}$ ) or the iNOS -/- (PCR product, $1288 \mathrm{bp}$ ), which was visualized as a mutant form. The PCR products were then separated on an agarose gel and products were visualized with ethidium bromide. In Figure 2 is shown a composite PCR that was run using tail clip DNA from both iNOS -/- and WT mice. Lanes 1-3, from iNOS -/- mice, show a single band that corresponds to the standard for iNOS -/-, shown in Lane 4 and Lane 13. DNA from WT mice is shown in Lanes 5-7; the PCR product is of a different size and corresponds to that found with a cDNA standard (data not shown). The samples in Lanes 8-12 are derived from tail clip DNA from WT mice probed with primer for the iNOS -/- to demonstrate that WT animals do not exhibit the iNOS -/- genotype.

\section{Ureteral Obstruction}

WT and iNOS -/- mice ( $n=6$ per group) underwent right unilateral ureteral ligation with 4-0 silk suture through an abdominal midline incision under sterile conditions. Animals were anesthetized with $0.12-0.15$ cc intraperitoneal pentobarbital $(6.5 \mathrm{mg} / \mathrm{ml})$. Shamoperated animals underwent identical operations, however with manipulation of the right ureter only. Animals were housed in groups of 3 to 5 and fed standard chow and water ad libitum. At 14 days, both obstructed and control kidneys were removed follow- 


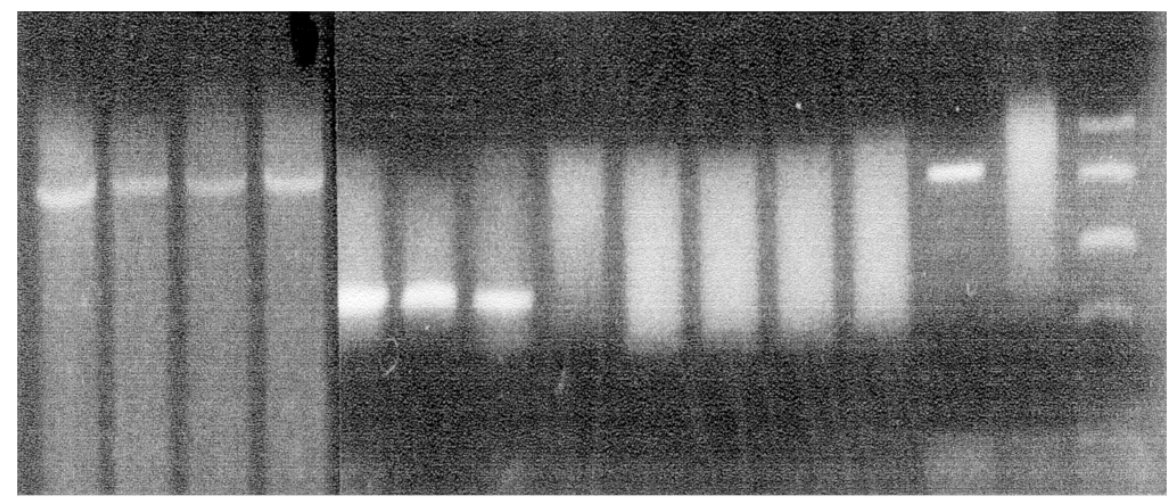

\section{$\begin{array}{lllllllllllllll}1 & 2 & 3 & 4 & 5 & 6 & 7 & 8 & 9 & 10 & 11 & 12 & 13 & 14 & 15\end{array}$}

Figure 2.

Confirmation of genotype in WT and iNOS -/- mice. Reactions were run using 70 ng of tail clip DNA as described in "Materials and Methods." The PCR product for WT is $413 \mathrm{bp}$; the PCR product of the iNOS -/- is $1288 \mathrm{bp}$. Samples: Lanes 1 to 3, tail clip DNA from three separate iNOS -/- mice; Lane 4, cDNA std, iNOS -/-; Lanes 5 to 7 , tail clip DNA from three separate WT mice probed with WT primers; Lanes 8 to 10, tail clip DNA from WT mice probed with iNOS -/- primers; Lane 13, cDNA std, iNOS -/-; Lane 14, PCR blank, no DNA; Lane 15, mw markers.

ing the administration of IP pentobarbital. Animal treatment adhered to approved institutional guidelines.

\section{Renal Morphology}

Sections of hemisected obstructed and contralateral kidneys were fixed in $10 \%$ neutral buffered formalin and embedded in paraffin. Sections 5- $\mu \mathrm{m}$ thick were stained with hematoxylin and eosin and Masson's trichrome for light microscopic analysis. Histologic studies were performed in a blinded fashion, with treatment group unknown to the examiner.

\section{Morphometric Analysis: Interstitial Volume}

Measurements of interstitial volume were undertaken using a point-counting technique as previously described (Wright et al, 1996). Ten consecutive, nonoverlapping fields were evaluated in both the cortex and medulla at $400 \times$ on each trichrome-stained section using a $10 \times 10$ grid-imprinted reticle. All points not contained within tubular cells or lumen, Bowman's capsule, or blood vessel were considered interstitial. This fraction correlated with relative interstitial volume, and was evaluated in a blinded fashion by two individuals. Results are expressed as the percentage of the measured area that represents interstitial space.

\section{Tissue Hydroxyproline}

Hydroxyproline was measured using the colorimetric assay of Reddy and Enwemeka (1996). This assay uses Ehrlich's reagent and chloramine-T and is read at $550 \mathrm{~nm}$. Tissues were lyophilized and results expressed per mg dry weight of tissue. Hydroxyproline is considered to represent $10 \%$ of the collagen, as determined by using collagen standards in this assay. Results are adjusted accordingly.

\section{TGF- $\boldsymbol{\beta}$ Measurements}

Mink lung epithelial cells (MLECs), which were transfected with a PAl-1 promoter driving expression of luciferase in a TGF- $\beta_{1}$ responsive manner, were used for analysis of TGF- $\beta_{1}$ activity as previously described (Miyajima et al, 2000a). These cells were a generous gift from Dr. Daniel Rifkin. Transfected MLEC cells were plated in 96-multiwell plate at $37^{\circ} \mathrm{C}$ in a humidified incubator. The TGF- $\beta_{1}$ in the test sample was activated with $1 \mathrm{~N} \mathrm{HCl}\left(1\right.$ hour at $4^{\circ} \mathrm{C}$ ), followed by equilibration with $1 \mathrm{~N} \mathrm{NaOH}$. After 3 hours, the medium was replaced with the activated test sample and incubated overnight at $37^{\circ} \mathrm{C}$. Cells were then lysed and luciferase reagent (Promega, Madison, Wisconsin) was added to the cell lysates. Luciferase activity was measured in a luminometer.

\section{Macrophage Identification and Quantification}

Expression of macrophages was evaluated using immunoperoxidase in paraffin sections, as previously described (Scherr et al 1999; Wright et al, 1996). We used a rat-monoclonal antibody to the F4/80 antigen (Caltag Laboratories, Burlingame, California), a murine macrophage-specific cellular membrane marker, in formalin-fixed sections. No specific antigen retrieval was required using this antibody in paraffin-fixed sections.

Sections were deparaffinized with Hemo-De for 30 minutes, rehydrated in increasing concentrations of ethanol, and washed three times in PBS for 10 minutes. Endogenous peroxidase was quenched for 30 minutes with $0.3 \% \mathrm{H}_{2} \mathrm{O}_{2} /$ methanol solution. After washes in filtered water and PBS, a blocking step was included using $5 \%$ rabbit serum in conjunction with the avidin and biotin blocking solutions (Vector Kit; Vector Laboratories, Burlingame, California) for a total of 30 minutes. Primary antibody $(\mathrm{Ab})$ was then applied 
(dilution 1:150) overnight at $4^{\circ} \mathrm{C}$. Mouse adsorbed, biotinylated rabbit anti-rat secondary $\mathrm{Ab}$ (Caltag; 1:100) was incubated for 30 minutes, as was tertiary Ab (Biogenex, San Ramon, California; 1:100), with washes in PBS between each new Ab application. Slides were developed using a 1- to 5-minute incubation with diaminobenzidine, and counterstained with $10 \%$ hematoxylin for 2 minutes. Macrophages were counted in 10 consecutive, non-overlapping highpower fields, and the results expressed as numbers of macrophages per high-power field.

\section{Statistical Analysis}

Interstitial volume and macrophage number comparison between obstructed and control kidneys was analyzed using ANOVA (STATPAK; Northwest Analytical, Portland, Oregon). Significant differences $(p<$ 0.05) were validated using least significant differences. Data are expressed as mean \pm SEM.

\section{References}

Akimitsu T, Gute DC, and Korthuis RJ (1995). Leukocyte adhesion induced by inhibition of nitric oxide production in skeletal muscle. J Appl Physiol 78:1725-1732.

Beck PL, Xavier R, Ezedi E, Mizoguchi E, Mashimo H, Bahn A, and Podolsky D (1999). Paradoxical roles of different nitric oxide synthase isoforms in colonic injury. Gastroenterology 116:A798 (abstract).

Bhagat $K$ and Vallance P (1996). Nitric oxide 9 years on. J R Soc Med 89:667-673.

Border WA and Noble NA (1994). Transforming growth factor beta in tissue fibrosis. N Engl J Med 331:1286-1292.

Bryk R and Wolff DJ (1998). Mechanism of inducible nitric oxide synthase inactivation by aminoguanidine and L-N6-(1iminoethyl)lysine. Biochemistry 37:4844-4852.

Bryk R and Wolff DJ (1999). Pharmacological modulation of nitric oxide synthesis by mechanism-based inactivators and related inhibitors. Pharmacol Ther 84:157-178.

Chen PY, St. John PL, Kirk KA, Abrahamson DR, and Sanders PW (1993). Hypertensive nephrosclerosis in the Dahl/Rapp rat: Initial sites of injury and effect of dietary L-arginine supplementation. Lab Invest 68:174-184.

De Nicola L, Thomson SC, Wead LM, Brown MR, and Gabbai FB (1993). Arginine feeding modifies cyclosporine nephrotoxicity in rats. J Clin Invest 92:1859-1865.

Diamond JR, Kees-Folts D, Ding G, Frye JE, and Restrepo NC (1994). Macrophages, monocyte chemoattractant peptide-1, and TGF-beta 1 in experimental hydronephrosis. Am J Physiol 266:F926-933.

Eddy AA (1996). Molecular insights into renal interstitial fibrosis (editorial). J Am Soc Nephrol 7:2495-2508.

Gordon S, Lawson L, Rabinowitz S, Crocker PR, Morris L, and Perry VH (1992). Antigen markers of macrophage differentiation in murine tissues. Curr Top Microbiol Immunol 181:1-37.

Gulmi FA, Felsen D, and Vaughan ED Jr (1998). Pathophysiology of urinary tract obstruction. In: Walsh PC, Retik AB, Vaughan ED Jr, and Wein AJ, editors. Campbell's urology, 7th ed. WB Saunders: Philadelphia.
Hierholzer C, Harbrecht B, Menezes JM, Kane J, MacMicking J, Nathan CF, Peitzman AB, Billiar TR, and Tweardy DJ (1998). Essential role of induced nitric oxide in the initiation of the inflammatory response after hemorrhagic shock. J Exp Med 187:917-928.

Hou J, Kato H, Cohen RA, Chobanian AV, and Brecher $\mathrm{P}$ (1995). Angiotensin II-induced cardiac fibrosis in the rat is increased by chronic inhibition of nitric oxide synthase. J Clin Invest 96:2469-2477.

Huang A, Palmer LS, Hom D, Valderrama E, and Trachtman $H(2000)$. The role of nitric oxide in obstructive nephropathy. J Urol 163:1276-1281.

Ishidoya S, Morrissey J, McCracken R, Reyes A, and Klahr S (1995). Angiotensin II receptor antagonist ameliorates renal tubulointerstitial fibrosis caused by unilateral ureteral obstruction. Kidney Int 47:1285-1294.

Kanwar S and Kubes P (1995). Nitric oxide is an antiadhesive molecule for leukocytes. New Horiz 3:93-104.

Katoh T, Takahashi K, Klahr S, Reyes AA, and Badr KF (1994). Dietary supplementation with L-arginine ameliorates glomerular hypertension in rats with subtotal nephrectomy. J Am Soc Nephrol 4:1690-1694.

Kettritz U, Semelka RC, Brown ED, Sharp TJ, Lawing WL, and Colindres RE (1996). MR findings in diffuse renal parenchymal disease. J Magn Reson Imaging 6:136-144.

Kim YM, Bombeck CA, and Billiar TR (1999). Nitric oxide as a bifunctional regulator of apoptosis. Circ Res 84:253-6.

Klahr S and Morrissey JJ (1998). The role of growth factors, cytokines, and vasoactive compounds in obstructive nephropathy. Semin Nephrol 18:622-632.

Kone BC (1997). Nitric oxide in renal health and disease. Am J Kidney Dis 30:311-333.

MacMicking JD, Nathan C, Hom G, Chartrain N, Fletcher DS, Trumbauer M, Stevens K, Xie QW, Sokol K, Hutchinson N, Chen H, and Mudgett JS (1995). Altered responses to bacterial infection and endotoxic shock in mice lacking inducible nitric oxide synthase Cell 81:641-650.

Mashimo H and Goyal RK (1999). Lessons from genetically engineered animal models. IV. Nitric oxide synthase gene knockout mice. Am J Physiol 277:G745-750.

McMillan K, Adler M, Auld DS, Baldwin JJ, Blasko E, Browne LJ, Chelsky D, Davey D, Dolle RE, Eagen KA, Erickson S, Feldman RI, Glaser CB, Mallari C, Morrissey MM, Ohlmeyer MH, Pan G, Parkinson JF, Phillips GB, Polokoff MA, Sigal NH, Vergona R, Whitlow M, Young TA, Devlin JJ (2000). Allosteric inhibitors of inducible nitric oxide synthase dimerization discovered via combinatorial chemistry. Proc Natl Acad Sci USA 97:1506-11.

Miyajima A, Chen J, Lawrence C, Ledbetter S, Pigato J, Lemer M, Stern J, Poppas DP, Vaughan ED Jr, and Felsen D (2000a). Antibody to TGF- $\beta$ ameliorates tubular apoptosis in unilateral ureteral obstruction. Kidney Int. 58:2301-2313.

Miyajima A, Chen J, Poppas DP, Vaughan ED Jr, and Felsen D (2000b). Role of nitric oxide in renal tubular apoptosis of unilateral ureteral obstruction. J Urol 163 (Suppl): 83(abstract).

Moore WM, Webber RK, Jerome GM, Tjoeng FS, Misko TP, and Currie MG (1994). L-N6-(1-iminoethyl)lysine: A selective inhibitor of inducible nitric oxide synthase. J Med Chem 37:3886-3888. 
Morrissey JJ, Ishidoya S, McCracken R, and Klahr S (1996). Nitric oxide generation ameliorates the tubulointerstitial fibrosis of obstructive nephropathy. J Am Soc Nephrol 7:22022212.

Numaguchi K, Egashira K, Takemoto M, Kadokami T, Shimokawa H, Sueishi K, and Takeshita A (1995). Chronic inhibition of nitric oxide synthesis causes coronary microvascular remodeling in rats. Hypertension 26:957-962.

Peters H, Border WA, and Noble NA (1999). L-arginine supplementation increases mesangial cell injury and subsequent tissue fibrosis in experimental glomerulonephritis. Kidney Int 55:2264-2273.

Peters H, Border WA, and Noble NA (2000). Angiotensin II blockade and low-protein diet produce additive therapeutic effects in experimental glomerulonephritis. Kidney Int 57: 1493-1501.

Qiu B, Mashimo H, Vallance B, Blennerrhassett $\mathrm{P}$, and Collins $S$ (1998). Susceptibility of mice with specific NOS gene deletions to experimental colitis. Gastroenterology 114: A1065 (abstract).

Reddy GK and Enewmeka S (1996). A simplified method for the analysis of hydroxyproline in biological tissues. Clin Biochem 29:225-229.

Reyes AA, Karl IE, Kissane J, and Klahr S (1993). L-arginine administration prevents glomerular hyperfiltration and decreases proteinuria in diabetic rats. J Am Soc Nephrol 4:1039-1045.

Reyes AA, Karl IE, Yates J, and Klahr S (1994). Low plasma and renal tissue levels of L-arginine in rats with obstructive nephropathy. Kidney Int 45:782-787.

Reyes AA, Martin D, Settle S, and Klahr S (1992). EDRF role in renal function and blood pressure of normal rats and rats with obstructive uropathy. Kidney Int 41:403-413.

Scherr DS, Vaughan ED Jr, Wei J, Chung M, Felsen D, Allbright R, and Knudsen BS (1999). BCL-2 and p53 expression in clinically localized prostate cancer predicts response to external beam radiotherapy. J Urol 162:12-17.
Schulsinger DA, Marion DN, Kim FY, Felsen D, Cartelli C, Gross SS, and Vaughan ED Jr (1997). The nitric oxide system is activated during late unilateral ureteral obstruction. J Urol 157(Suppl.):209 (abstract).

Sharma AK, Mauer SM, Kim Y, and Michael AF (1993). Interstitial fibrosis in obstructive nephropathy. Kidney Int 44:774-788.

Sigusch HH, Campbell SE, and Weber KT (1996). Angiotensin II-induced myocardial fibrosis in rats: Role of nitric oxide, prostaglandins and bradykinin. Cardio Res 31:546-554.

Tomita H, Egashira K, Ohara Y, Takemoto M, Koyanagi M, Katoh M, Yamamoto H, Tamaki K, Shimokawa H, and Takeshita A (1998). Early induction of transforming growth factor-beta via angiotensin II type 1 receptors contributes to cardiac fibrosis induced by long-term blockade of nitric oxide synthesis in rats. Hypertension 32:273-9.

Trachtman H, Futterweit S, Garg P, Reddy K, and Singhal PC (1996). Nitric oxide stimulates the activity of a $72-\mathrm{kDa}$ neutral matrix metalloproteinase in cultured rat mesangial cells. Biochem Biophys Res Comm 218:704-708.

Wright EJ, McCaffrey TA, Robertson AP, Vaughan ED Jr, and Felsen D (1996). Chronic unilateral ureteral obstruction is associated with interstitial fibrosis and tubular expression of transforming growth factor-beta. Lab Invest 74:528-537.

Zeiher AM, Fisslthaler B, Schray-Utz B, and Busse R (1995). Nitric oxide modulates the expression of monocyte chemoattractant protein 1 in cultured human endothelial cells. Circ Res 76:980-986.

Zhang X, Xu X, Nasjletti A, and Hintze TH (2000). Amlodipine enhances NO production induced by an ACE inhibitor through a kinin-mediated mechanism in canine coronary microvessels. J Cardiovasc Pharmacol 35:195-202. 